\title{
Exercise-induced changes in interleukin-10 in patients with knee osteoarthritis: new perspectives?
}

\author{
Willem F Lems* and Debby den Uyl \\ See related research by Helmark et al., http://arthritis-research.com/content/12/4/R126
}

\begin{abstract}
Osteoarthritis (OA) of the knee is a common chronic disease leading to increased morbidity and reduced quality of life. Although exercise therapy has been shown to be beneficial for both pain and physical functioning, its underlying mechanism is not fully understood. However, a recent study found an exercise-induced increase in interleukin-10 levels, to which anti-inflammatory and chondroprotective properties are ascribed, in the (peri-)synovial fluid of patients with knee OA. These interesting results provide more insight into the effects of exercise in $\mathrm{OA}$ and need to be validated and confirmed. Hopefully, the study offers a promising basis for further research.
\end{abstract}

Osteoarthritis (OA) of the knee is a common chronic disease affecting more than half of older persons. Moreover, owing to both aging and life-style factors, its prevalence is expected to increase in the coming years. Undoubtedly, OA may lead to increased morbidity and reduced quality of life in many older people. OA progresses slowly and for clinicians it is a well-known phenomena that, although patients with OA suffer from chronic pain, exacerbations may occur and these are associated with more pain, low-grade inflammation, and joint swelling.

Unfortunately, therapeutic options that interfere with the progression of OA have not yet been found. For that reason, pain reduction with analgesic and antiinflammatory drugs and life-style interventions, such as weight loss in adipose older persons and physical therapy, are the treatment options for OA. Exercise therapy has

*Correspondence: wf.lems@vumc.nl

VU University Medical Centre, Department of Rheumatology, De Boelelaan 1117, 1081 HV, Amsterdam, The Netherlands been shown to be beneficial for pain and physical functioning. However, the underlying mechanism of the effects of exercise therapy has not been fully elucidated.

From that perspective, we welcome the article by Helmark and colleagues [1] in this issue of Arthritis Research \& Therapy. In the article, which shows that exercise therapy appears to influence the intra-articular inflammatory response, the authors observed the effects of exercise on (peri-)synovial biochemical markers by using the microdialysis method. Surprisingly, this study showed that exercise might also influence the inflammatory processes in the joint. Women with symptomatic knee OA and a Kellgren-Lawrence grade of at least 1 $(\mathrm{n}=31)$ were randomly assigned to nonexercise or exercise therapy. Directly after exercise, blood and urine samples were taken and microdialysis catheters were placed. The catheters were positioned intra-articularly and extra-articularly (near the synovium), and every 30 minutes, samples were collected over a period of 3 hours. The intra-articular and peri-synovial interleukin-10 (IL-10) levels were significantly increased after exercise, whereas no changes were found in IL-10 levels in the nonexercise group. Levels of IL-6, IL-8, and tumor necrosis factor-alpha (TNF- $\alpha)$ were significantly increased in both groups.

Elevated levels of several cytokines, such as IL-6, IL-1, and TNF- $\alpha$, are found in early and advanced OA [2]. Low-grade inflammation has been implicated in the pathogenesis of OA, and synovitis is suggested to predict progression $[3,4]$. In line with this, there are several experimental studies that showed that neutralizing the inflammatory response resulted in prevention of both chondrocyte apoptosis and cartilage degradation $[5,6]$. Increased levels of IL-10 have been identified earlier in synovial fluid of patients with OA [2]. However, the changes that exercise brought about in IL-10 levels, as shown in this study [1], are novel and interesting findings.

Exercise has been shown to protect against cartilage loss in animal studies [7], and recently exercise therapy 
has also been shown to influence human cartilage properties $[8,9]$. For example, shortly after exercise, an elevation of serum levels of cartilage oligomeric matrix protein (COMP) was found in patients with OA, suggesting an effect on cartilage metabolism [8]. These interesting new results allow speculation that exercise therapy might have direct effects on cartilage homeostasis by affecting the intra-articular cytokine levels [1]. The anti-inflammatory and the recently shown antiapoptotic effects of IL-10 on chondrocytes suggest that IL-10 might have chondroprotective activities [10]. However, minimal evidence of alterations in cartilage markers could be found in this study. Intra-articular COMP decreased instantly after exercise $(P<0.05)$, but no changes in other cartilage degradation markers, such as COMP serum levels or CTX-II (type II collagen) urine levels, were found; such changes would have made the evidence stronger. It should be noted that owing to the technique, the authors were limited in the amount of synovial material available, and it was not possible to investigate more markers. Why there were no significant changes in serum levels is not fully clear, but that might be related to the fact that serum levels reflect cartilage damage of all joints.

The observation of increased intra-articular IL-10 levels after exercise therapy could lead to a greater understanding of the role of exercise in knee OA. Relevant new research questions arise: Why is IL-10 upregulated after exercise? Could the increase in IL-10 and decrease in COMP be confirmed in comparable studies? What is the evidence for a causal relationship of IL-10 and COMP, or are the changes coincidences? Why is COMP, but none of the other cartilage degradation markers, decreased?

Given the paucity of effective treatment strategies in OA, these observations hopefully will prompt further investigations on the influence of exercise and intraarticular IL-10 levels and cartilage degradation. Nevertheless, the first step is that these data be validated and confirmed by others.

\section{Conclusions}

Exercise therapy influences the intra-articular IL-10 levels in patients with knee OA. The pathophysiological mechanism underlying the effects of exercise therapy has not been fully elucidated. It has been suggested that exercise therapy results in cartilage degradation by affecting the low-grade inflammatory state. Further research is required.

\section{Abbreviations}

COMP, cartilage oligomeric matrix protein; IL, interleukin; OA, osteoarthritis; TNF-a, tumor necrosis factor-alpha.

\section{Competing interests}

The authors declare that they have no competing interests.

\section{Published: X Month 2010}

\section{References}

1. Helmark IC, Mikkelsen UR, Børglum J, Rothe A, Petersen MCH, Andersen O, Langberg $H$, Kjaer M: Exercise increases interleukin-10 levels both intraarticularly and peri-synovially in patients with knee osteoarthritis: a randomized controlled trial. Arthritis Res Ther 2010, 12:R126.

2. Loria MP, Dambra P, Moretti B, Patella V, Capuzzimati L, Cavallo E, Nettis E, Pesce V, Dell'Osso A, Simone C, Tursi A: Role of cytokines in gonarthrosis and knee prosthesis aseptic loosening. J Orthop Sci 2004, 9:274-279.

3. Ayral X, Pickering EH, Woodworth TG, Mackillop N, Dougados M: Synovitis: a potential predictive factor of structural progression of medial tibiofemoral knee osteoarthritis -- results of a 1 year longitudinal arthroscopic study in 422 patients. Osteoarthritis Cartilage 2005, 13:361-367.

4. Conaghan PG, D'Agostino MA, Le Bars M, Baron G, Schmidely N, Wakefield R, Ravaud P, Grassi W, Martin-Mola E, So A, Backhaus M, Malaise M, Emery P, Dougados M: Clinical and ultrasonographic predictors of joint replacement for knee osteoarthritis: results from a large, 3-year, prospective EULAR study. Ann Rheum Dis 2010, 69:644-647.

5. Bar-Yehuda S, Rath-Wolfson L, Del Valle L, Ochaion A, Cohen S, Patoka R, Zozulya G, Barer F, Atar E, Piña-Oviedo S, Perez-Liz G, Castel D, Fishman P. Induction of an antiinflammatory effect and prevention of cartilage damage in rat knee osteoarthritis by CF101 treatment. Arthritis Rheum 2009, 60:3061-3071.

6. Weng LH, Wang CJ, Ko JY, Sun YC, Su YS, Wang FS: Inflammation induction of Dickkopf-1 mediates chondrocyte apoptosis in osteoarthritic joint. Osteoarthritis Cartilage 2009, 17:933-943.

7. Otterness IG, Eskra JD, Bliven ML, Shay AK, Pelletier JP, Milici AJ: Exercise protects against articular cartilage degeneration in the hamster. Arthritis Rheum 1998, 41:2068-2076.

8. Andersson ML, Thorstensson CA, Roos EM, Petersson IF, Heinegard D, Saxne T: Serum levels of cartilage oligomeric matrix protein (COMP) increase temporarily after physical exercise in patients with knee osteoarthritis. BMC Musculoskelet Disord 2006, 7:98.

9. Roos EM, Dahlberg L: Positive effects of moderate exercise on glycosaminoglycan content in knee cartilage: a four-month, randomized, controlled trial in patients at risk of osteoarthritis. Arthritis Rheum 2005, 52:3507-3514.

10. Schulze-Tanzil G, Zreiqat H, Sabat R, Kohl B, Halder A, Muller RD, John T: Interleukin-10 and articular cartilage: experimental therapeutical approaches in cartilage disorders. Curr Gene Ther 2009 Aug 1. [Epub ahead of print].

doi:10.1186/ar3084

Cite this article as: Lems WF, den Uyl D: Exercise-induced changes in interleukin-10 in patients with knee osteoarthritis: new perspectives? Arthritis Research \& Therapy 2010, 12:131. 